\title{
Cognitive Burden Estimation for Visuomotor Learning with fNIRS
}

\author{
David R.C. James, Felipe Orihuela-Espina, Daniel R. Leff, George P. Mylonas, \\ Ka-Wai Kwok, Ara W. Darzi, Guang-Zhong Yang \\ Imperial College London, United Kingdom \\ \{d.james, f.orihuela-espina, d. leff, george.mylonas\} aimperial.ac.uk \\ \{k.kwok07,a.darzi,g.z.yang\}@imperial.ac.uk
}

\begin{abstract}
Novel robotic technologies utilised in surgery need assessment for their effects on the user as well as on technical performance. In this paper, the evolution in 'cognitive burden' across visuomotor learning is quantified using a combination of functional near infrared spectroscopy (fNIRS) and graph theory. The results demonstrate escalating costs within the activated cortical network during the intermediate phase of learning which is manifest as an increase in cognitive burden. This innovative application of graph theory and fNIRS enables the economic evaluation of brain behaviour underpinning task execution and how this may be impacted by novel technology and learning. Consequently, this may shed light on how robotic technologies improve human-machine interaction and augment minimally invasive surgical skills acquisition. This work has significant implications for the development and assessment of emergent robotic technologies at cortical level and in elucidating learning-related plasticity in terms of inter-regional cortical connectivity.
\end{abstract}

Keywords: Robotic surgery, cognitive burden, graph theory, near infrared spectroscopy, neuroergonomics.

\section{Introduction}

The use of robots for enhancing surgical performance, particularly for minimally invasive surgery (MIS) has opened a number of new opportunities. In addition to tremor filtration, motion scaling, and virtual fixtures (active constraints), it also provides a natural framework for integrating pre- and intra-operative imaging combined with augmented reality. Recently, the concept of gaze-contingent motor channelling (GCMC) has been proposed as a way of potentially improving hand-eye coordination for certain aspects of surgery where movement impacts on the surgical site, e.g. beating heart surgery or targeted therapy and focused energy delivery of robotically assisted MIS [1]. The method relies on eye gaze and its dynamic pursuit movement to form constraints that can effectively direct hand movements for accurate targeting in the presence of large tissue deformation. It is intuitive that in robotic tasks where surgeons are effectively 'perceptually docked' with their environment [2], subjectspecific neurocognitive behaviour plays a critical role that influences the final quality

T. Jiang et al. (Eds.): MICCAI 2010, Part III, LNCS 6363, pp. 319-326, 2010.

(C) Springer-Verlag Berlin Heidelberg 2010 
of the procedure. The paradigm of neuroergonomics utilizes neuroimaging to assess the impact of undertaking tasks at brain level and uses this information to aid the development of performance enhancing tools [3]. Methods of assessing task-induced mental workload are directed towards the amount of mental resources required versus those available i.e. 'functional segregation' [4]. However, this approach is unable to capture interactions between recruited cortical areas, i.e. 'functional integration.' The concept of 'cognitive burden' relates to the most efficient use of cortical interactions and deviations thereof. Neuroplasticity underpins the learning process [5], therefore it is necessary to study the evolution of learning related cortical interactions over time.

The purpose of this paper is to evaluate economic changes in a cortical network associated with the acquisition of a complex simulated beating heart procedure with and without the use of GCMC. At each phase of learning, the activated network is evaluated to assess the degree of task-induced cognitive burden.

Functional Near Infrared Spectroscopy (fNIRS) [6, 7] allows for the assessment of brain function in a more realistic human-robotic environment. The prefrontal cortex (PFC) and parietal cortex (PC) are interrogated since a frontoparietal (F-P) network underlies visuomotor tracking [8]. Task evoked brain activation is identified using a classical statistical approach, leading to the construction of an activity guided map. A cortical network defined by phase locked cooperation between channels is analysed in terms of its economy within the graph theory framework to unveil longitudinal fluctuations in task related cognitive burden. In this paper, a novel use of graph theory in the analysis of fNIRS data enables appreciation of the interaction between cortical regions subserving task execution. Ensuing information may be valuable in ensuring that novel technology is beneficial to the user and that performance improvements are not detrimental to other facets of task execution. This study may have potential implications for guiding the design and use of performance enhancing technology not only in surgery but in other safety critical tasks such as aviation. It is hypothesized that performance improvements evoked by GCMC will be secondary to enhanced interaction within a F-P network.

\section{Methods}

\subsection{Subjects, Task Paradigm and fNIRS}

A total of 21 healthy right-handed subjects were recruited to track a moving target on a simulated beating heart using a virtual tool controlled by a haptic manipulator (SensAble Tech. MA). Participants performed this without (control group, $n=11$ ) or with GCMC (experiment group, $\mathrm{n}=10$ ). An eyetracking device (Tobii, Sweden) distinguishes the subject's fixation point. This information is used to define the force required to constrain the subject's hand allowing accurate target localization as illustrated in Fig. 1 [1]. A block design experiment was conducted comprising baseline rest (30s), followed by five blocks of the task (20s) and inter trial rest (30s). Performance was determined as the distance from the tool tip to the target (pixels). Subjects undertook task sessions on six separate days. Cortical haemodynamic data was acquired with a fNIRS system (ETG-4000, Hitachi Medical Corp, Japan). Twenty four channels in two 3 x 3 arrays were positioned over the left PC and PFC using the UI 

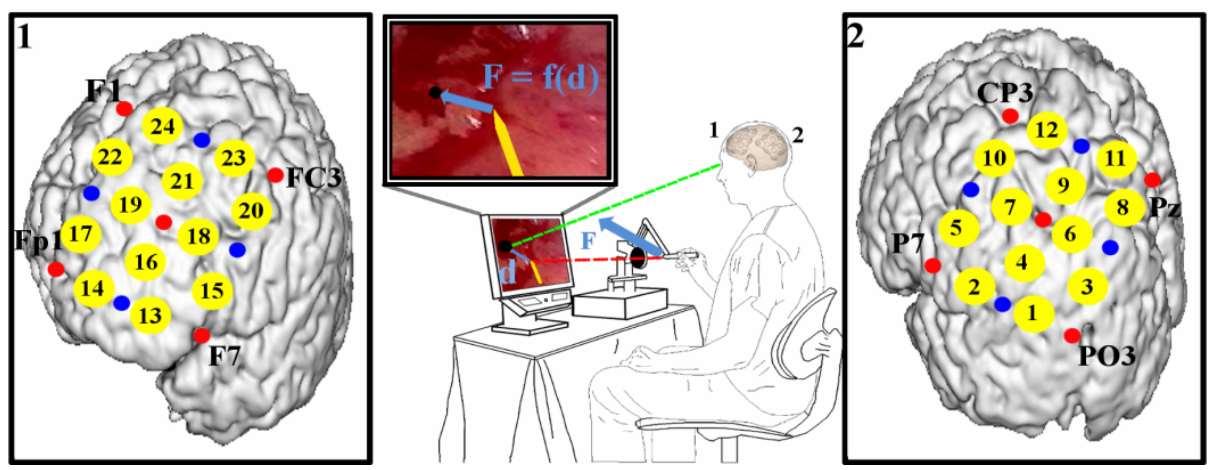

Fig. 1. Experimental set up (central panel) illustrating the moving target (black dot) and the virtual tool (yellow line) controlled by the haptic manipulator. A force $(\mathrm{F})$ proportional to the distance (d) from the tool tip to the fixation point is fed back to the subject (via the haptic manipulator) to diminish this separation [1]. The movement space of the haptic phantom was restricted to $2 \mathrm{D}$ as the monitor screen is inherently a $2 \mathrm{D}$ space. Optode arrays can be appreciated on the subject's head and the approximate channel location overlain onto a reference atlas for PFC and PC (subplots 1 and 2 respectively). Channels (yellow circles) and optode emitters and detectors (red and blue circles respectively) are displayed in relation to the UI 10/10 coordinate systems [9].

10-10 system [9] as illustrated in Fig. 1. Raw optical data was converted into relative changes in oxygenated haemoglobin $\left(\triangle \mathrm{HbO}_{2}\right)$ and deoxygenated haemoglobin ( $\triangle H H b$ ) using the modified Beer-Lambert Law, then decimated, linearly detrended, and subjected to data integrity checks. Representative haemodynamic data are demonstrated in Fig. 2. Task induced $\triangle \mathrm{HbO}_{2}$ and $\mathrm{AHHb}$ (t-test rank sign, $\alpha<5 \%$ ) were determined for each session and channel for each group.

\subsection{Cross Correlation and Activity Guided Network Formation}

Let $\Delta \mathrm{Hb}_{i}$ and $\triangle \mathrm{Hbj}$ be the bidimensional $\left.<\Delta \mathrm{HbO}_{2}, \Delta \mathrm{HHb}\right\rangle$ haemodynamic response at channels $i$ and $j$ respectively and $T_{i}$ and $T_{j}$ their corresponding signal length in samples. Let $R_{i, j}\left(\tau_{t}, \tau_{H b}\right)$ be the normalised 2D cross-correlation between the responses at channels $i$ and $j$, where $\tau_{t}$ is the temporal lag and $0 \leq \tau_{t}<T_{i}+T_{j}-1$ and $\tau_{H b}$ is the haemodynamic dimensional lag and $0 \leq \tau_{H b}<3$. An $m \times m$ phase locked zero lagged correlation matrix $C=\left\{c_{i j}=R_{i, j}\left(\tau_{t}=\left(T_{i}+T_{j}-1\right) / 2, \tau_{H b}=1\right)\right\}$ can be formed between each pair of channels. A pseudo-metric of signal similarity can be constructed as $d_{i j}=1-R_{i, j}$, and the accompanying distance matrix $d=\left\{d_{i, j}\right\}$. The fNIRS channels can be regarded as nodes in a graph. A fully connected weighted undirected graph $G(C)$ can be constructed using $c_{i j}$ as the weight of the connecting edge between channels $i$ and $j$, and $d_{i j}$ representing the ease of information flow between the channels. The lower the $d_{i j}$, the easier to transmit information between nodes $i$ and $j . G(C)$ is then pruned retaining only activated channels as defined below. The haemodynamic activity signature as recorded by fNIRS is described as a task-evoked increase in $\mathrm{HbO}_{2}$ 
and a concomitant decrease in $H H b$. Each haemoglobin species can vary in either direction with respect to the pre-stimulus baseline, and any change may or may not reach the statistical threshold. The following four patterns describe cortical activity by means of a t-test $\left(\alpha<5 \%\right.$ ): (A) $\triangle \mathrm{HbO}_{2}$ increment and $\triangle \mathrm{HHb}$ decrement both reaching statistical significance, (B and C) $\triangle \mathrm{HbO}_{2}$ increment and $\triangle \mathrm{HHb}$ decrement with only one reaching significance (D) $\triangle \mathrm{HbO}_{2}$ increment and $\triangle \mathrm{HHb}$ decrement with neither species reaching significance. A threshold was then applied to the connected graph, $G(C)$ permitting only those links between any two channels representing the activity patterns (A-D), thereby capturing the evolving relationships within an activated F-P network. It is customary to modulate the strength of the activity guided edges according to the statistical significance of the pattern at each of the edge endings. Here we use scales $1,0.75,0.75$ and 0.5 for patterns $A$ to D respectively, thus:

$$
\begin{aligned}
& \widehat{C}=\left\{c_{i j} \cdot \text { scaleFactor }_{i} \cdot \text { scaleFactor }_{j}\right\} \\
& \widehat{D}=\left\{d_{i j} \cdot \text { scaleFactor }_{i} \cdot \text { scaleFactor }_{j}\right\}
\end{aligned}
$$

The activity guided brain network is then $G(\widehat{C})$. This serves as the substrate for calculating the measures of network cost and efficiency.
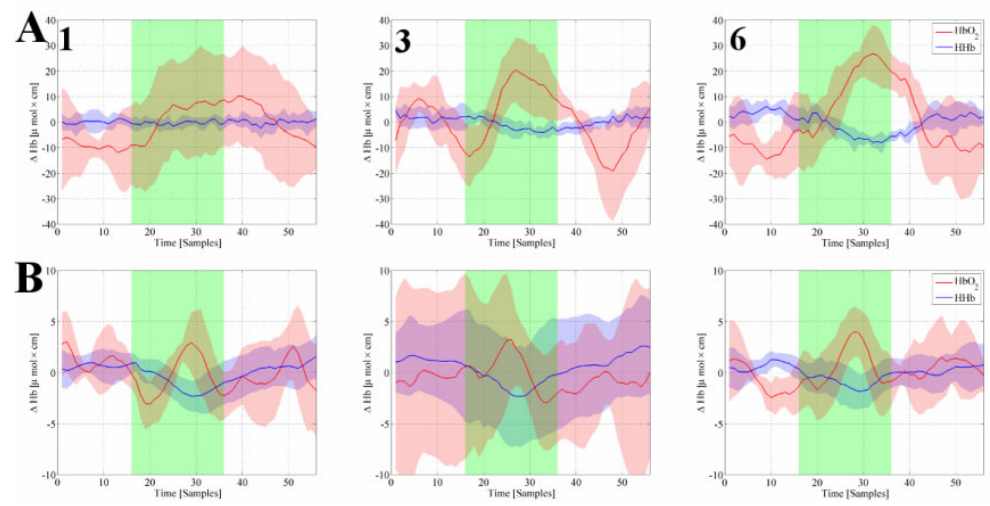

Fig. 2. Session averaged haemodynamic data from representative control (A) and GCMC (B) subjects for sessions 1, 3 and 6. Mean (bold line) and standard deviation (shaded region) are indicated for both $\mathrm{HbO}_{2}$ (red) and $\mathrm{HHb}$ (blue). The 20s task period (green shaded region) is bounded by rest and recovery periods (white).

\subsection{Network Efficiency and Cognitive Burden}

Excluding self connections (intra-channel) for which the flow of information between neurons is assumed infinite, the cost of a weighted graph or network is defined as per Eq. 3, and the network global efficiency has been defined as in Eq. 4 [10]:

$$
K(G)=\sum_{i \in G} \sum_{j \in G} d_{i j \mid i \neq j}
$$




$$
E_{g l o b}(G)=\frac{1}{N(N-1)} \sum_{i \in G} \sum_{j \in G} \frac{1}{d_{i j \mid i \neq j}}
$$

Both quantities, cost and global efficiency are normalised in proportion to the maximum efficiency and cost of a comparable network comprising all possible connections [11]. The economy of a cortical network has been defined as:

$$
\operatorname{Economy}(G)=E_{\text {glob }}^{\text {norm }}(G)-K^{\text {norm }}(G)
$$

From its definition, it is intuitive that the cognitive burden is inversely related to the network economy. Furthermore, if the cost dominates the equation, the cognitive burden will be positive and high. Conversely, if the efficiency dominates the equation, the cognitive burden will be negative and low.

\section{Results}

One subject withdrew from the study after the second session and the remaining 20 subjects completed all six sessions conducted over a median of eight days. Fig. 3 illustrates the evolution in F-P excitation across the experiment for gaze-assisted and unassisted learners.

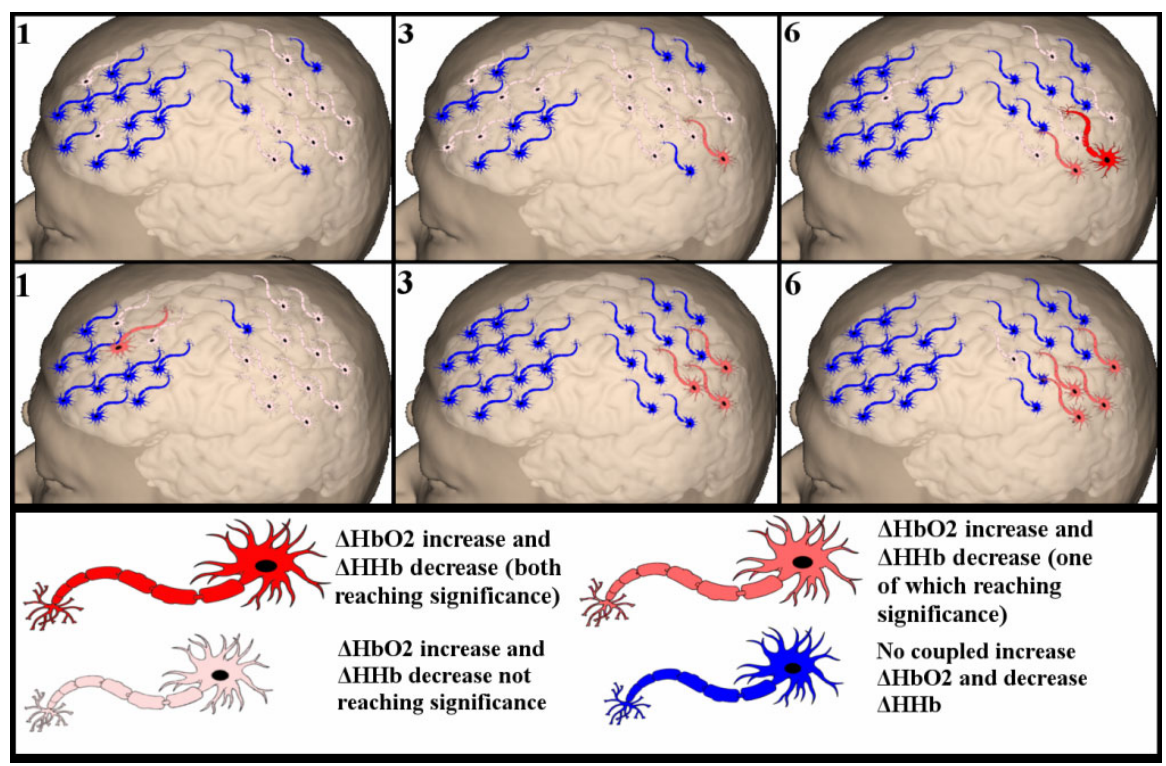

Fig. 3. Groupwise statistical analysis of cortical haemodynamic responses in unassisted (top) and GCMC-assisted (bottom) learners across practice (Sessions 1, 3 and 6). Channels are represented by coloured neurons according to the represented haemodynamic pattern (figure key). The spatial attenuation in PFC and PC responses and magnification of activity in the PPC can be appreciated. 

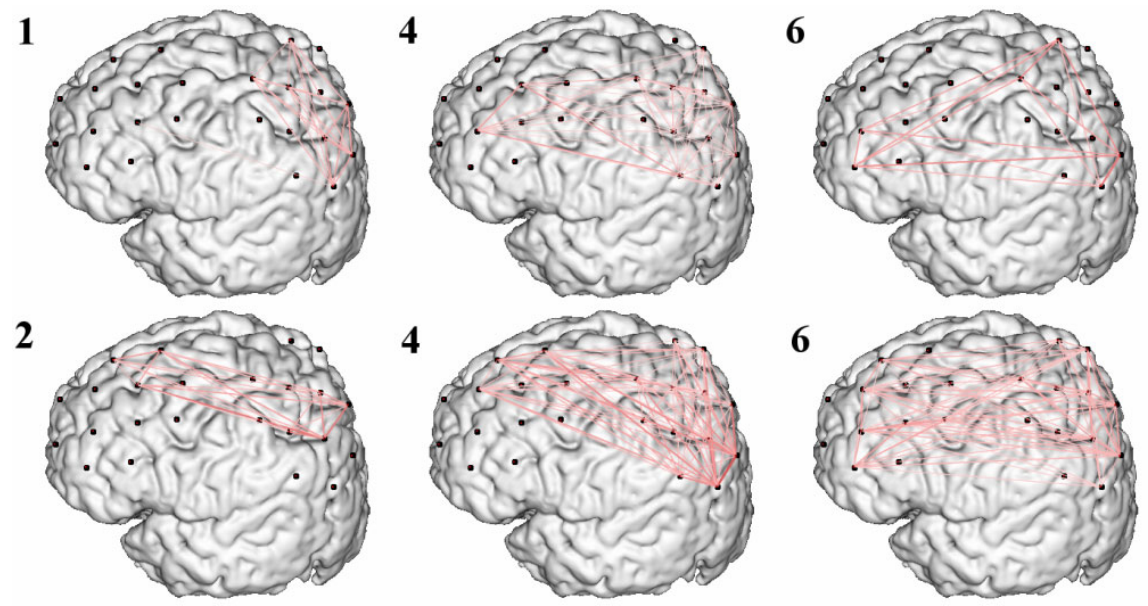

Fig. 4. F-P activated network graphs overlaid onto an MRI image of the cortical surface. Graph nodes (black circles) indicate approximate channel location and connections are weighted according to the strength of the association between nodes. A representative control (top) and GCMC (bottom) subject is illustrated at practice session time points (labelled black). The majority of connections are formulated around the intermediate phase of practice.

Longitudinal changes in brain behaviour may be appreciated as a spatial attenuation in the F-P response and a magnification of excitation within the posterior parietal cortex (PPC). These longitudinal changes appear to occur more rapidly in GCMC enabled learners. Fig. 4 displays the evolution in regional connectivity within the activated F-P network for GCMC-assisted and unassisted learners. During the intermediate phase of learning, a greater number of intra- and inter-regional functional connections are evident, which may reflect formation of new cortical networks. In general, at each phase, a greater number of cortical connections were observed in assisted learners, reflecting the impact of GCMC assistance on F-P connectivity. Fig. 5 depicts the progression of the cost, efficiency and therefore cognitive burden evoked by the task at each time point across learning. Both groups progressed from a region of low cost and low efficiency (session 1) to a region of low cost but increased efficiency (session 6). This is achieved with different network development strategies. Evidently, an escalation in activated network cost accompanies the functional increase in cortical connections during intermediate learning. Furthermore, the magnitude of this cost increase is greater in GCMC- assisted learners, and occurs just after the sessions associated with maximal improvement in technical skill in this group $\left(1^{\text {st }}\right.$ session accuracy (pixels) median $(\mathrm{SD})=46.3(22.4) ; 2^{\text {nd }}$ session $=30.2(15.8)$ $(p<0.000)$, and significantly better accuracy compared to the control group until practice termination (session 3-6, $p=0.019,0.023,0.001,0.000$ ). In contrast to the changes observed in early practice, a reduction in the cognitive burden and an increase in F-P network efficiency seem to define ongoing performance enhancement in late practice (sessions 3-6). 

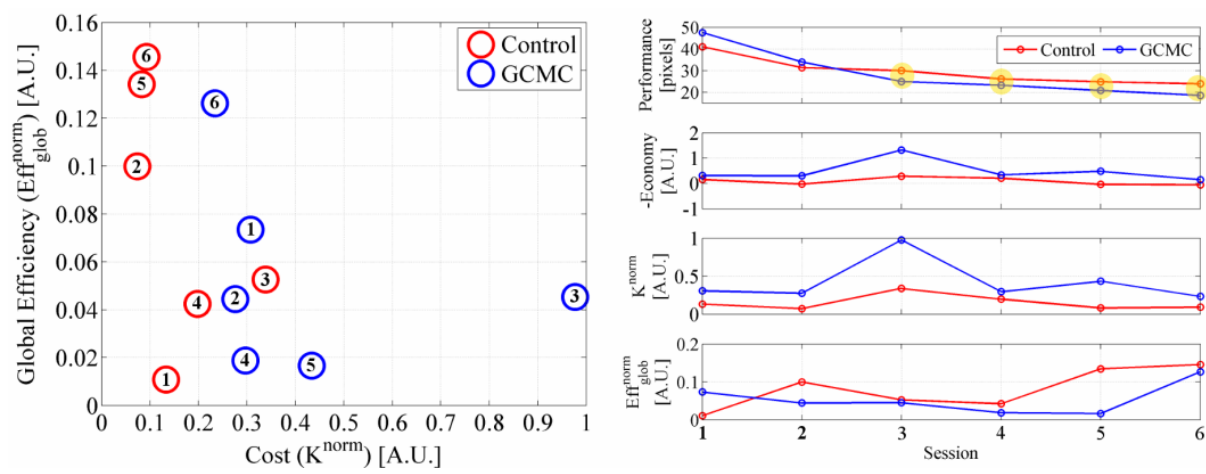

Fig. 5. Evolution in the cognitive burden across practice for control (red) and GCMC (blue). The cost efficiency plane (left panel) demonstrates the progression of these parameters across practice sessions (1-6) and that the foundation for the increase in cognitive burden demonstrated in session 3 (right panel) is due to an increase in cost. By session 6 both groups have migrated to a region indicative of a high efficiency and low cost. GCMC users significantly outperformed the control group in session 3-6 (highlighted yellow).

\section{Discussion and Conclusion}

In this paper, we have proposed a novel framework for analysing longitudinal fNIRS data in order to estimate the cognitive burden associated with learning visuomotor tracking. Using this strategy, it has been possible to unveil transformations within an activated F-P network as a result of deliberate practice. Enhanced tracking accuracy is indexed by a functional increase in cognitive burden and attenuation in cortical haemodynamics across a F-P network. More importantly, fundamental differences in the pattern of cortical excitation change and variation in cognitive burden have been exposed depending upon the mode of skills learning. Specifically, GCMC learners demonstrate the greatest increase in network connections and cognitive burden, and more rapid F-P attenuation. Therefore, the technical improvements induced by GCMC over and above unaided learning may be secondary to improved F-P communication. This may have relevance to training on tasks that require precise visuomotor skill and also in the assessment of emergent surgical technologies.

Acknowledgements. The authors would like to thank Daniel Ramirez-Cano for useful discussions.

\section{References}

1. Mylonas, G.P., Kwok, K.W., Darzi, A., Yang, G.Z.: Gaze-contingent motor channelling and haptic constraints for minimally invasive robotic surgery. In: Med. Image Comput. Comput. Assist. Interv. Int. Conf., vol. 11, pp. 676-683 (2008)

2. Yang, G.Z., Mylonas, G.P., Kwok, K.W., Chung, A.: Perceptual docking for robotic control. In: Dohi, T., Sakuma, I., Liao, H. (eds.) MIAR 2008. LNCS, vol. 5128, pp. 21-30. Springer, Heidelberg (2008) 
3. Parasuraman, R.: Neuroergonomics: research and practice. Theoretical Issues in Ergonomics Science 4, 5-20 (2003)

4. Just, M.A., Carpenter, P.A., Miyake, A.: Neuroindices of cognitive workload: neuroimaging, pupillometric and event-related potential studies of brain work. Theoretical Issues in Ergonomics Science 4, 56-88 (2003)

5. Kelly, A.M., Garavan, H.: Human functional neuroimaging of brain changes associated with practice. Cereb Cortex 15, 1089-1102 (2005)

6. Leff, D.R., Elwell, C.E., Orihuela-Espina, F., Atallah, L., Delpy, D.T., Darzi, A.W., Yang, G.Z.: Changes in prefrontal cortical behaviour depend upon familiarity on a bimanual coordination task: an fNIRS study. Neuroimage 39, 805-813 (2008)

7. Leff, D.R., Orihuela-Espina, F., Atallah, L., Darzi, A., Yang, G.Z.: Functional near infrared spectroscopy in novice and expert surgeons-a manifold embedding approach. In: Med. Image Comput. Comput. Assist. Interv. Int. Conf., vol. 10, pp. 270-277 (2007)

8. Hill, H., Raab, M.: Analyzing a complex visuomotor tracking task with brain-electrical event related potentials. Human Movement Science 24, 1-30 (2005)

9. Jurcak, V., Tsuzuki, D., Dan, I.: 10/20, 10/10, and 10/5 systems revisited: their validity as relative head-surface-based positioning systems. Neuroimage 34, 1600-1611 (2007)

10. Latora, V., Marchiori, M.: Efficient behaviour of small-world networks. Physical Review Letters 87 (2001)

11. Achard, S., Bullmore, E.: Efficiency and cost of economical brain functional networks. PLoS Computational Biology 3, 174-183 (2007) 